\title{
Ultrafast Electronic Energy Transfer Beyond the Weak Coupling Limit in a Proximal but Orthogonal Molecular Dyad
}

\author{
Gordon J. Hedley, ${ }^{\top}$ Arvydas Ruseckas, ${ }^{\top}$ Andrew C. Benniston, ${ }^{\S}$ Anthony Harriman ${ }^{\S}$ * ${ }^{*}$ and Ifor D. \\ W. Samuel ${ }^{\top}, *$ \\ 'Organic Semiconductor Centre, SUPA, School of Physics and Astronomy, University of St Andrews, North Haugh, \\ St Andrews, Fife, KY16 9SS, United Kingdom \\ $\S$ Molecular Photonics Laboratory, School of Chemistry, Bedson Building, Newcastle University, Newcastle upon \\ Tyne, NE1 7 RU, United Kingdom \\ KEYWORDS: Fluorescence. Photophysics . Förster theory . Oligothiophene . BODIPY dyes.
}

\begin{abstract}
Electronic energy transfer (EET) from a donor to an acceptor is an important mechanism that controls the light harvesting efficiency in a wide variety of systems, including artificial and natural photosynthesis and contemporary photovoltaic technologies. The detailed mechanism of EET at short distances or large angles between the donor and acceptor is poorly understood. Here the influence of the orientation between the donor and acceptor on EET is explored using a molecule with two nearly perpendicular chromophores. Very fast EET with a time constant of 120 fs is observed, which is at least forty times faster than the time predicted by Coulombic coupling calculations. Depolarization of the emission signal indicates that the transition dipole rotates through ca. $64^{\circ}$, indicating the near orthogonal nature of the EET event. The rate of EET is found to be similar to structural relaxation rates in the photo-excited oligothiophene donor alone, which suggests that this initial relaxation brings the dyad to a conical intersection where the excitation jumps to the acceptor.
\end{abstract}

\section{INTRODUCTION}

Electronic energy transfer (EET) is known to play significant roles in numerous natural (e.g., photosynthesis, photolyase enzymes, DNA damage) and artificial (e.g., light harvesting antenna, advanced signaling protocols, organic solar cells) processes. ${ }^{1-5}$ This realization has led to a tremendous resurgence of interest in the generic mechanisms that underpin EET in both solid and liquid phases, ${ }^{6,7}$ fueled by recent observations of energy transfer without spectral overlap in single-molecule fluorescence experiments ${ }^{8}$ and the possible involvement of quantum coherence. ${ }^{9}$ Most notable among the emerging research trends has been the detailed examination of EET across short (i.e., <30 Å) separation distances:;0-12 apart from fundamental interest, such events are likely to be significant in light-harvesting arrays, ${ }^{13}$ organic photovoltaics and OLEDs. ${ }^{14}$ The rate of EET in such systems can be crucial in defining their overall efficiencies. Fast rates of EET are desirable to out-compete loss mechanisms and so maximize the EET efficacy.

Long-range EET is well described by conventional Förster theory based on a point-dipole approximation, ${ }^{15}$ however, this theory does not hold in cases where the donor/acceptor separation is comparable to the sum of the transition dipole moment vectors divided by the electronic charge. ${ }^{16}$ Modest improvements are achieved by modified Förster theory which takes into account charge densities. Likewise, super-exchange interactions, being closely linked to electron transfer, cannot explain the rates of EET at short distances. ${ }^{17}$ Hybrid approaches, based primarily on modified Redfield theory, ${ }^{18}$ do account for EET between closely spaced donor-acceptor pairs in specific cases but a universal theory for such circumstances is lacking. Very fast EET is generally enabled when there is good spectral overlap between the donor and acceptor or strong electronic coupling between the chromophores. Poor spectral overlap or weak electronic coupling are thus not desirable when aiming for fast EET. A further restriction on the rate of EET is the mutual orientation of transition dipole moment vectors associated with the donor and acceptor, although the internal directions of these vectors might be more difficult to delineate from the better defined molecular geometry. Application of Förster theory requires knowledge of the mutual alignment of these vectors and also their directions with respect to the molecular axis. When the transition dipole moment vectors are aligned perpendicular to each other, ${ }^{19}$ and especially if one of these is orthogonal to the molecular axis, ${ }^{10,20}$ significant extra challenges are presented to fully explain resonant energy transfer between closely spaced chromophores.

Here, we have observed the rate of EET in a molecular dyad where the donor, this being an oligothiophene unit, and acceptor, this being a boron dipyrromethene (Bodipy) dye, are covalently linked and held at near orthogonal geometries. ${ }^{21}$ In this particular molecular system, we find that EET is exceptionally fast, with a time constant of 120 fs. 
This transfer time is observed both with the decay of donor emission and the rise of acceptor emission. Fluorescence anisotropy is used to track the emission dipole orientation, where we find that the dipole rotates by ca. $65^{\circ}$ with respect to the excitation polarization. This angle is in good agreement with that derived from single crystal diffraction and molecular modelling. The observed time constant for EET is forty times faster than the fastest rate that can be found with resonant energy transfer theoretical treatments. We explore the possible mechanisms to explain such fast EET, and find that ultrafast relaxation in the oligothiophene agrees well with the rate of EET, indicating that such relaxation can enable the excited state to reach a position on the potential energy surface where a conical intersection with the Bodipy surface enables crossing to its electronic manifold.

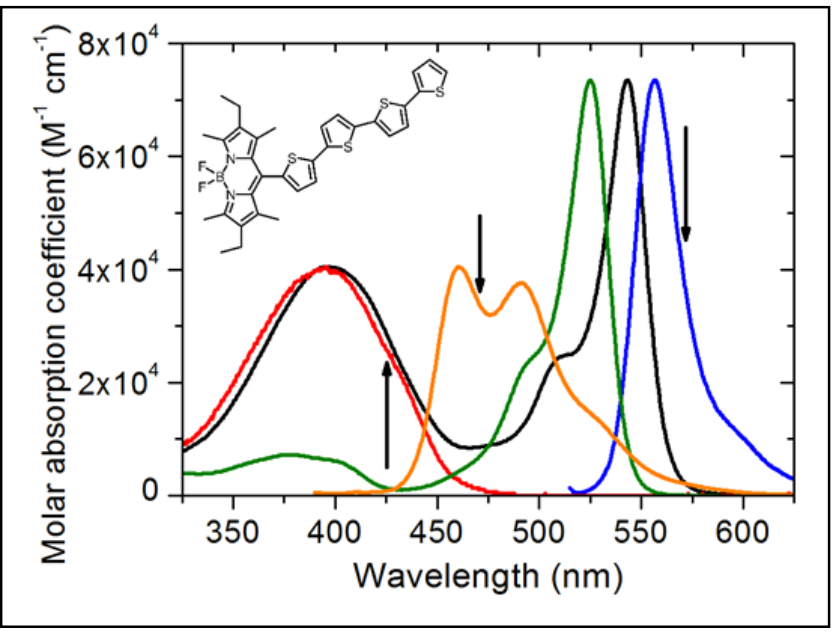

Figure 1. Absorption (black curve) and emission (blue curve) spectra for BOD-T4 with the chemical structure given as an inset. Absorption (red) and emission (orange) spectra of $\mathrm{T}_{4}$, together with the absorption (green) spectrum of Bodipy are also shown. The arrows represent the excitation wavelength $(425 \mathrm{~nm})$ and the two emission detection wavelengths (470 and $570 \mathrm{~nm}$ ). Computational studies indicate that the transition dipole moment vectors follow the long molecular axes for the isolated control compounds and also for the full dyad. It should be mentioned, however, that these calculations might not fully reflect the consequences of any intramolecular charge-transfer effects.

\section{MATERIALS and METHODS}

Samples were available from earlier work ${ }^{19}$ and were further purified immediately before use. Steady-state emission spectra were recorded with a Jobin-Yvon Fluorolog tau-4 instrument equipped with an Oxford Instruments Optistat DN cryostat. Energy-minimized geometries were optimized at the B3LYP/6-311G** level with TURBOMOLE and used for subsequent calculations. Transition density cube data were generated for the entire molecule with volume elements of $0.02 \AA^{3}$. For laser spectroscopy, the dyad was dissolved in spectroscopic grade cyclohexane, the limited solubility of the material ensuring that concentrations were kept below $0.1 \mathrm{mg} / \mathrm{ml}$. Measurements were made in a rotating cuvette (optical path length of $0.5 \mathrm{~mm}$ ) to minimize degradation. Ultrafast luminescence measurements were performed using up-conversion spectroscopy. ${ }^{22}$ The $850 \mathrm{~nm}$ output (FWHM = $100 \mathrm{fs}$ ) from a Ti:Sapphire oscillator was frequency doubled with a $\mathrm{BBO}$ crystal to generate the excitation pulse, while residual $850 \mathrm{~nm}$ light was used as the gating pulse after reflection with an optical delay line. Fluorescence was collected and focused onto a sumfrequency generation (SFG) BBO crystal along with the 850 $\mathrm{nm}$ gating pulse. The SFG light (typically $300-330 \mathrm{~nm}$ ) was spatially and spectrally filtered before being detected with a photomultiplier tube.

Fluorescence anisotropy measurements were completed with the use of a Berek compensator, rotating the polarization of the excitation, while the detection polarization was kept fixed. For magic angle measurements, the excitation polarization was set at $54.7^{\circ}$ with respect to detection. For anisotropy studies, the excitation polarization was rotated parallel and perpendicular to the detection. A temporal shift exists between the two polarizations which will alter the calculated anisotropy at early times; Raman signals from water indicated that emission from perpendicular excitation arrived 38 fs earlier than that from parallel excitation. All anisotropy data were corrected for this shift prior to analysis. The instrument response function was also deduced from the water Raman signals and found to possess a full-width at half maximum (FWHM) of 270 fs. The gfactor for the anisotropy measurements was taken as unity, since the detection polarization was kept fixed for both excitation polarizations while rotation of the excitation polarization gave no loss of pump intensity.

\section{RESULTS}

The studied molecular dyad comprises near orthogonally linked chromophores of oligothiophenes (TN), these being important components of many organic field effect transistors, and a Bodipy dye connected via a single $\mathrm{C}-\mathrm{C}$ bond (Figure 1). The near perpendicular alignment of the respective transition dipole moment vectors presents a further challenge to theory when attempting to calculate the rates of EET. The main target dyad, BOD-T4, has a tetrathiophene $\left(\mathrm{T}_{4}\right)$ donor but confirmatory studies were made with the corresponding pentathiophene $\left(\mathrm{T}_{5}\right)$ derivative, although this compound has limited solubility. Single crystal diffraction patterns ${ }^{21}$ for BOD-T4 show that the two subunits are held in an orthogonal geometry with the dipyrrin nucleus adopting a dihedral angle of $89.5^{\circ}$ with the $\mathrm{T}_{4}$ unit, with a center-to-center separation of 9.3 Å. Quantum chemical calculations predict a dihedral angle of $88.7^{\circ}$ between the dipyrrin plane and the first thiophene unit but highlight structural bending and twisting along the $\mathrm{T}_{4}$ unit that breaks the molecular symmetry. ${ }^{23}$ The crucial feature, however, relates to the mutual orientation of the respective transition dipole moment vectors, which follow the long 
molecular axis in each case. Structural distortions that position $\mathrm{T}_{4}$ above or below the Bodipy plane, for which the calculations indicate an average out-of-line angle of $28^{\circ}$, or rotations around the connecting $\mathrm{C}-\mathrm{C}$ bond do not affect orthogonality of these vectors. In contrast, lateral translation of the entire $\mathrm{T}_{4}$ donor with respect to the acceptor, leading to loss of the T-shaped geometry, would facilitate throughspace EET. ${ }^{24}$ This distortive motion, together with modest curvature of the $\mathrm{T}_{4}$ unit, reduces the angle between the transition dipole moment vectors to $72^{\circ}$ in the most extreme geometry.

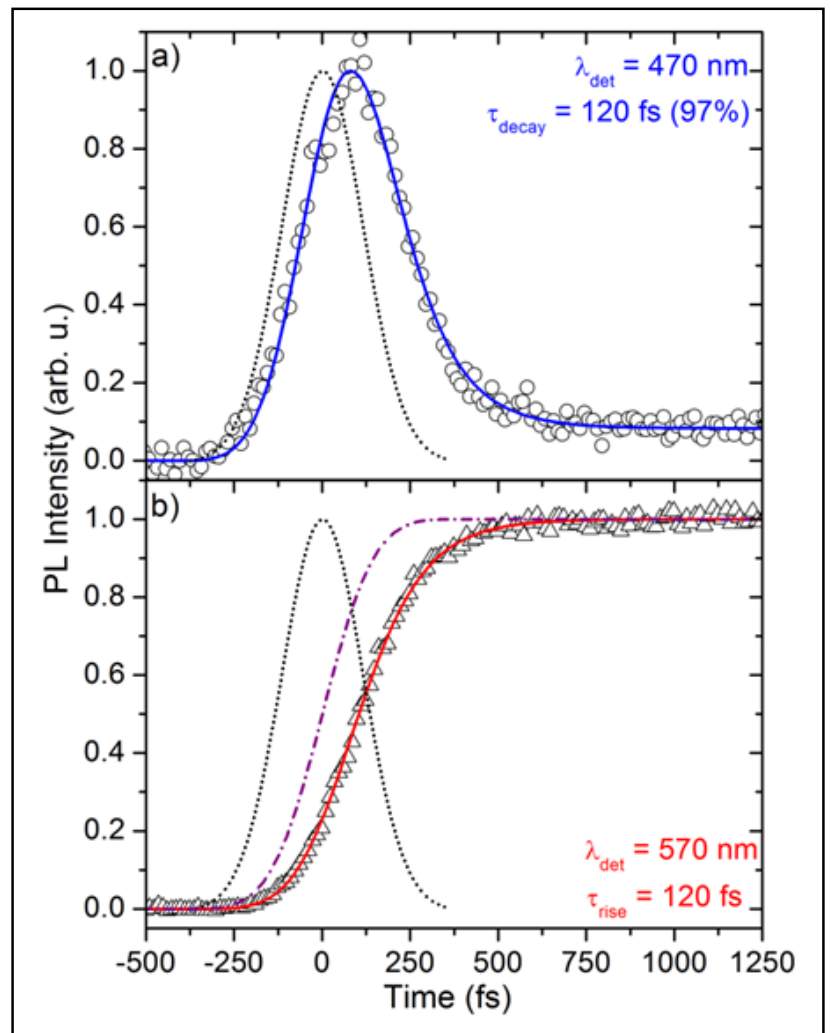

Figure 2. Ultrafast photoluminescence of BOD-T4 in cyclohexane measured at the magic angle $\left(54 \cdot 7^{\circ}\right)$. Excitation was at $425 \mathrm{~nm}$ and emission was measured at (a) $470 \mathrm{~nm}$ corresponding to $\mathrm{T}_{4}$ emission and (b) $570 \mathrm{~nm}$ corresponding to Bodipy emission. The data are the open symbols, the instrument response functions the dotted lines (270 fs FWHM) and the fits the solid lines with a decay time and rise-time in (a) and (b) respectively of $120 \pm 10 \mathrm{fs}$. The purple dot-dashed line in (b) shows the response which would be recorded for an instantaneous rise, indicating that the observed PL formation occurs after the pulse.

The absorption and photoluminescence (PL) spectra of the dyad in cyclohexane solution are shown in Figure 1. When the dyad is dissolved in cyclohexane, light-induced electron transfer is unlikely to compete with intramolecular EET but absorption transitions associated with $\mathrm{T}_{4}\left(\lambda_{\mathrm{MAX}}\right.$ $\left.=396 \mathrm{~nm} ; \varepsilon_{\mathrm{MAX}}=40,500 \mathrm{M}^{-1} \mathrm{~cm}^{-1}\right)$ and Bodipy $\left(\lambda_{\mathrm{MAX}}=543\right.$ $\mathrm{nm} ; \varepsilon_{\mathrm{MAX}}=73,500 \mathrm{M}^{-1} \mathrm{~cm}^{-1}$ ) are well resolved. At $425 \mathrm{~nm}$, approximately $95 \%$ of incident photons are absorbed by $\mathrm{T}_{4}$ while the spectral overlap integral $\left(J_{D A}=7.6 \times 10^{-5} \mathrm{~cm}\right)$ for emission from $\mathrm{T}_{4}$ and absorption by Bodipy is relatively weak. Fluorescence from the dyad occurs with a quantum yield $\left(\Phi_{\mathrm{F}}\right)$ of $0.24 \pm 0.005$ and lifetime $\left(\tau_{\mathrm{S}}\right)$ of $1.3 \pm 0.05 \mathrm{~ns}$, these being comparable to data collected for a control compound lacking the $\mathrm{T}_{4}$ unit. ${ }^{25}$ More importantly, cursory comparison with the appropriate $\mathrm{T}_{4}$ control $\left(\Phi_{\mathrm{F}}=0.18\right.$ $\left.\pm 0.002, \tau_{\mathrm{S}}=0.52 \pm 0.03 \mathrm{~ns}\right)$ shows that fluorescence from the $\mathrm{T}_{4}$ unit in BOD-T 4 is essentially absent $\left(\Phi_{\mathrm{F}}<10^{-4}, \tau_{\mathrm{S}}<30\right.$ ps). Furthermore, comparison of the fluorescence excitation spectrum of BOD-T4 with the absorption spectrum is fully consistent with quantitative EET from $\mathrm{T}_{4}$ to Bodipy under these conditions (see Supporting Information).

Using much improved temporal resolution with illumination at $425 \mathrm{~nm}$, thereby preferentially exciting $\mathrm{T}_{4}$ in the dyad, it becomes possible to monitor the progress of EET along the molecular axis. Figure 2 shows the fluorescence dynamics at a detection wavelength of $470 \mathrm{~nm}$, representing $\mathrm{T}_{4}$ emission, and at $570 \mathrm{~nm}$, representing Bodipy fluorescence. At $470 \mathrm{~nm}$, a fast decay component is observed, while at $570 \mathrm{~nm}$ a fast but finite rise is recorded. Iterative reconvolution of a trial function comprising an exponential term with the instrument response function (IRF) enables the best-fit parameters of the exponential to be deduced. It is found that both the decay at $470 \mathrm{~nm}$ and the rise at 570 $\mathrm{nm}$ give a satisfactory fit to a time constant of $120 \pm 10 \mathrm{fs}$, with a small $(<3 \%)$ offset accompanying the $470 \mathrm{~nm}$ decay. The rise-time measured for the appearance of Bodipy fluorescence at $570 \mathrm{~nm}$ appears inconsistent with the signal evolving within the IRF. This latter comparison shows clearly that the rise of fluorescence at $570 \mathrm{~nm}$ is measurably slower than the IRF, indeed the full amplitude of the $120 \mathrm{fs}$ rise component is required to fit the formation dynamics.

$$
r=\frac{I_{\|}-I_{\perp}}{I_{\|}+2 I_{\perp}}
$$

The direct evidence obtained from looking at the emission dynamics of the two chromophores gives a strong indication that EET from $\mathrm{T}_{4}$ to Bodipy occurs with a time constant of 120 fs. To provide additional support, and to solicit information about the orientations of the two chromophores, we measured the fluorescence anisotropy. Electronic energy transfer from $\mathrm{T}_{4}$ to Bodipy is expected to lead to a rotation of the emission dipole by an angle similar to that derived from the structural analyses. This change in emission dipole orientation should manifest itself in the anisotropy of the emission; that is, the intensity of the emission detected with polarization parallel and perpendicular to the polarization of the excitation laser field. The anisotropy factor, r, is defined ${ }^{26}$ according to Equation 1, where $I_{\perp}$ and $I_{\perp}$ are the fluorescence intensities parallel and perpendicular to the excitation beam. For a rod-like emitter, a value of $r=0.4 \pm 0.03$ indicates a dipole lying parallel to the excitation polarization, while a value of $r=-0.2 \pm 0.01$ 
is obtained if the emission dipole lies perpendicular to excitation. ${ }^{27}$ Emission is normally recorded at an angle of $54.7^{\circ}$ to the excitation polarization, the so-called "magic angle" which nullifies polarization effects, and indeed was used for the data presented in Figure 2.

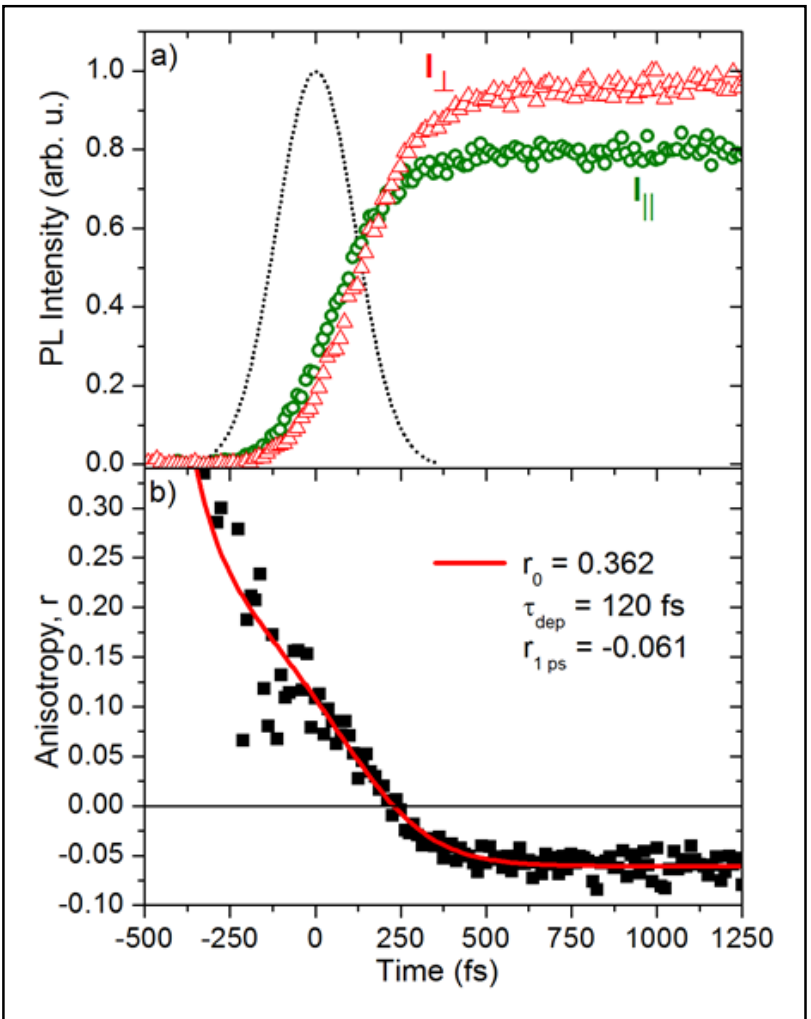

Figure 3. Fluorescence polarization dynamics in BOD$\mathrm{T}_{4}$ with $425 \mathrm{~nm}$ excitation and $570 \mathrm{~nm}$ detection. a) Emission detected parallel ( $\mathrm{I}_{\mid}$- open circles) and perpendicular ( $\mathrm{I}_{\perp}$ - open triangles) to the polarization of the excitation laser field, with the IRF shown as the dotted line. b) Calculated anisotropy $(\mathrm{r}$ ) of the data from the top panel according to equation 1 in the main text. Fitting of the anisotropy kinetics is with the auto-reconvolution method as described in the text and Supporting Information, with best fit parameters as shown.

To record emission parallel and perpendicular to excitation, we used a Berek compensator to rotate the polarization of the excitation beam with respect to the detector. Figure 3 shows the emission kinetics for BOD-T4 when exciting at $425 \mathrm{~nm}$ and measuring emission from Bodipy at $570 \mathrm{~nm}$, both parallel and perpendicular to the excitation polarization. In the upper panel, the directly recorded data are shown, indicating that parallel emission starts stronger but perpendicular emission becomes larger after $250 \mathrm{fs}$. In the lower panel, the anisotropy calculated using Equation 1 is presented, showing evolution of the emission dipole orientation with respect to time. Fitting of the anisotropy dynamics is achieved with deconvolution of the IRF using the auto-reconvolution technique ${ }^{28}$ (see Supporting Information for full details). The fit gives an initial value for the anisotropy of $r_{0}=0.36 \pm 0.03$ and decays to a final value of $-0.06 \pm 0.005$ with a time constant of $120 \pm 10$ fs due to reorientation of the transition dipole. This result agrees very well with the previously mentioned decay and rise-times of $120 \pm 10 \mathrm{fs}$.

$$
\alpha=\cos ^{-1}\left(\sqrt{\frac{5 r+1}{3}}\right)
$$

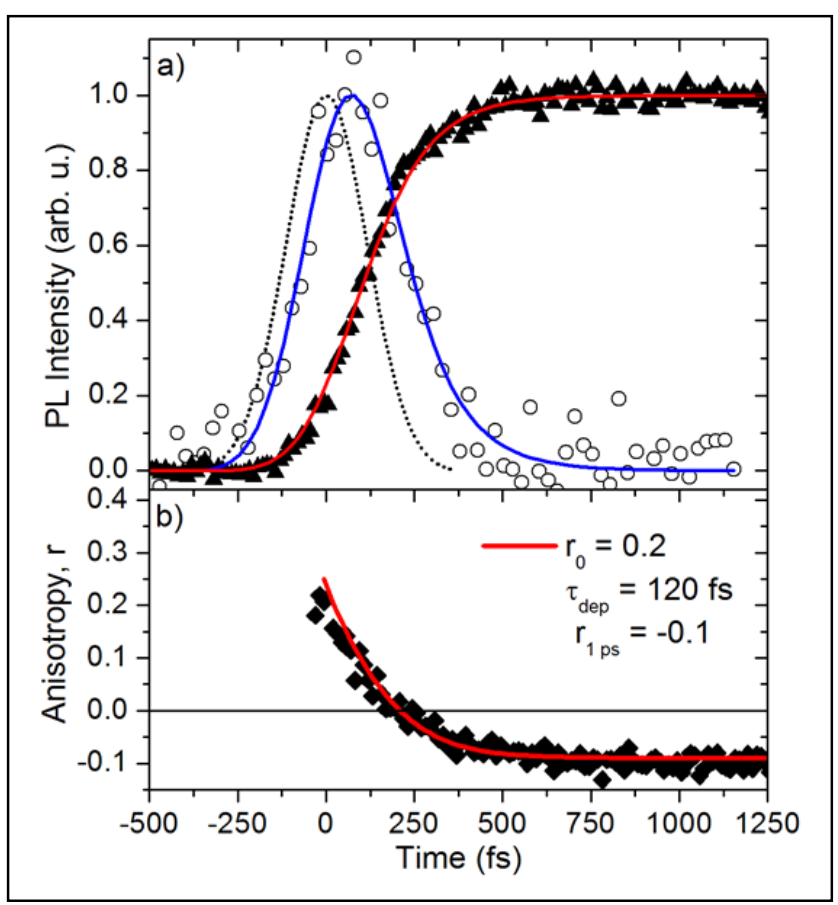

Figure 4. Fluorescence and its anisotropy kinetics for the BOD-T 5 dyad. a) Detected magic angle emission at $470 \mathrm{~nm}$ (open circles) and $570 \mathrm{~nm}$ (solid triangles), representing $\mathrm{T}_{5}$ and Bodipy emission respectively. The solid lines are fits to the data, and a time constant of $120 \pm 10$ fs (decay at $470 \mathrm{~nm}$ and rise-time at $570 \mathrm{~nm}$ ) is found to fit best to the data. The IRF is shown as the dotted line. b) Fluorescence anisotropy kinetics at $570 \mathrm{~nm}$ detection, calculated according to equation 1. Fitting is performed with the auto-reconvolution method, and the best fit parameters are as shown.

Anisotropy can be a powerful descriptor of orientations in the molecular dyad and additional information can be extracted beyond the time constant for depolarization. The final anisotropy value, $\mathrm{r}_{\text {inf }}$, is $-0.06 \pm 0.005$, and can be related $^{27}$ to an angle of orientation with respect to excitation through Equation 2, where $\alpha$ is the angle and $r$ is the anisotropy. Directly calculated for $\mathrm{r}=-0.06 \pm 0.005$ this gives an $\alpha$ value of $61^{\circ}$, however due consideration has to be given to the small amount (i.e., ca. $5 \%$ ) of direct absorption by the second-excited singlet state $\left(\mathrm{S}_{2}\right)$ state of Bodipy at $425 \mathrm{~nm}$. With this in mind, we measured the amount of 
depolarization that accompanies internal conversion for the isolated Bodipy chromophore when exciting the $\mathrm{S}_{\mathrm{o}} \rightarrow$ $\mathrm{S}_{2}$ transition and monitoring at $570 \mathrm{~nm}\left(\mathrm{~S}_{1} \rightarrow \mathrm{S}_{0}\right)$ (see Supporting Information). At times $>1$ ps, after internal conversion to $S_{1}$ is complete, the anisotropy is $0.23 \pm 0.01$. Armed with this information, together with the fractional molar absorption coefficients at $425 \mathrm{~nm}$, we conclude that the corrected angle between the respective transition dipole moments is modified slightly to $64^{\circ}$. Agreement with the computed geometry, allowing for the nonlinear shape of $\mathrm{T}_{4}$, is considered to be good.

The investigation was extended to include a similar dyad but equipped with a more extended donor, namely $\mathrm{T}_{5}\left(\Phi_{\mathrm{F}}\right.$ $\left.=0.32 \pm 0.005, \tau_{\mathrm{S}}=0.85 \pm 0.05 \mathrm{~ns}\right)$, which limits solubility but, because of increased $\pi$-conjugation, helps separate $\mathrm{T}_{5}$ absorption $\left(\lambda_{\mathrm{MAX}}=417 \mathrm{~nm}, \varepsilon_{\mathrm{MAX}}=44,500 \mathrm{M}^{-1} \mathrm{~cm}^{-1}\right)$ from upper-lying transitions associated with Bodipy. The same set of measurements was completed as for BOD- $\mathrm{T}_{4}$ and the results are presented in a condensed form in Figure 4. The time constant for EET from $\mathrm{T}_{5}$ to Bodipy at $120 \pm 10 \mathrm{fs}$ is the same as found for BOD-T4. Thus, the addition of the extra thiophene unit has no observable effect on the rate of EET, despite the significantly improved spectral overlap integral $\left(\mathrm{J}_{\mathrm{DA}}=1.51 \times 10^{-4} \mathrm{~cm}\right)$ between $\mathrm{T}_{5}$ emission and Bodipy absorption. Interestingly, the anisotropy, where the initial $r_{o}$ value of 0.2 is consistent with the higher fractional absorption by the donor, reaches a limiting value of $r_{\text {inf }}=-0.1 \pm$ 0.01 at longer times. This is somewhat lower than found for BOD-T 4 and does not require correction for direct $\mathrm{S}_{0}$ $\mathrm{S}_{2}$ absorption by Bodipy. The corresponding angle between the respective transition dipole moment vectors is now calculated to be $66^{\circ}$ compared to a value of $68^{\circ}$ deduced from molecular modelling. Here, the derived angles are in excellent agreement.

\section{DISCUSSION}

We have been able to unambiguously observe that EET through a reorientation of ca. $65^{\circ}$ in the BOD-T 4 dyad occurs with a time constant of $120 \pm 10$ fs. This extremely fast EET from the near perpendicularly orientated oligothiophene donor to the Bodipy acceptor is a surprising and novel result. Understanding of the mechanisms that can enable such fast EET is a pressing question in the field of chemical physics. Indeed, EET can broadly be divided into three regimes; namely, strong, intermediate and weak coupling. ${ }^{29}$ The interplay between electronic coupling among the chromophores and coupling of the excitation to its environment determines the nature of the energy-transfer event. In the weak-coupling regime, the environmental reorganization energy is significantly larger than the electronic coupling energy, and so EET proceeds with a deterministic "random walk". If, on the other hand, the electronic coupling is significantly larger than the reorganization energy then the two states mix, and the excitation oscillates between the two in a quantum mechanical manner, with phase information being preserved. A third case ex- ists, however, where the electronic coupling and reorganization energy are of similar magnitude, and thus a combination of the two processes occurs where excitation phase information is preserved while energy transfer takes place but interactions with the environment destroy the phase over time.

Several factors suggest to us that EET in these dyads is not subject to the Förster weak-coupling limit. Fast EET across orthogonally sited chromophores held in close proximity is not unknown but presents a challenge to contemporary theory. In general, rates of EET appear too fast to be explained within the weak coupling regime of Förster theory, ${ }^{18}$ although it should be stressed that there is a scarcity of experimental data from which to compose new models. Thus, the measured rate constant $\left(\mathrm{k}_{\mathrm{EET}}=8 \pm 0.7 \times 10^{12} \mathrm{~s}^{-1}\right)$ is roughly $1,000-$ fold higher than that calculated on the basis

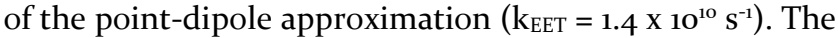
extended line-dipole approach ${ }^{30}$ does not achieve the observed rate $\left(\mathrm{k}_{\mathrm{EET}}=1.4 \times 10^{11} \mathrm{~s}^{-1}\right)$ nor does replacement of the dipole moment vector with changes in transition dipole moment associated with individual atoms ${ }^{31}$ localized on donor and acceptor $\left(\mathrm{k}_{\mathrm{EET}}=2.1 \times 10^{11} \mathrm{~s}^{-1}\right)$. The computed rate constant, even for the full transition density cube approach introduced by Fleming and co-workers ${ }^{32}$ remains greatly inferior $\left(\mathrm{k}_{\mathrm{EET}}=1.1 \times 10^{11} \mathrm{~s}^{-1}\right)$ to the measured value. These various calculations used the energy-minimized geometry with spectroscopic properties calculated for the relaxed excited states of donor and acceptor. Small fluctuations around this geometry have only minor effects on the calculated $k_{\mathrm{EET}}$ value. ${ }^{10}$ Each of these approaches predicts that EET will be ca. $50 \%$ faster for $\mathrm{BOD}_{4} \mathrm{~T}_{4}$ than BOD-T5, mainly because of the extended length of the latter, but this is not seen experimentally.

We thus turn our attention to the excited state processes that influence the dyad's interaction with the environment, and so govern EET beyond the weak-coupling limit where electronic coupling may be of similar strength. To this end, the dyad was studied in methylcyclohexane (MCHX) at $77 \mathrm{~K}$, at this temperature MCHX forms a glass. Steady-state emission spectroscopy shows that fluorescence from $\mathrm{T}_{4}$ in the dyad remains very weak, with an extremely small emission peak at $22,290 \mathrm{~cm}^{-1}$. Excitation and absorption spectra remain identical, indicating that quantitative EET must still be occurring at low temperature. The absorption spectrum associated with the $\mathrm{T}_{4}$ unit is better resolved at $77 \mathrm{~K}$ indicating a higher population of planar conformers in the glassy matrix. The more challenging, but essential, experiment relates to the profile of the weak $\mathrm{T}_{4}$ emission at $293 \mathrm{~K}$ as recorded for extremely pure samples of BOD- $\mathrm{T}_{4}$ in $\mathrm{MCHX}$. Here, the o-o transition lies at $22,415 \mathrm{~cm}^{-1}$, which is barely (i.e., $125 \mathrm{~cm}^{-1}$ ) red-shifted from that observed at $77 \mathrm{~K}$. It is found, therefore, that the emission at both $293 \mathrm{~K}$ and $77 \mathrm{~K}$ arises from a planar $\mathrm{S}_{1}$ state whereas the ground state comprises a more heterogeneous ensemble. This is consistent with a substantial geometry change (i.e., planarization) after excitation, 33 which is also evidenced by the poor mirror symmetry and a large Stokes 
shift for $\mathrm{T}_{4}$ (and also $\mathrm{T}_{5}$ ) emission at room temperature (Figure 1).

Previous work observed that sub-picosecond planarization can occur in longer oligothiophenes with hexyl side chains. ${ }^{34}$ We examined this situation in our tetrathiophene oligomers by looking at ultrafast time-resolved emission on the red-side of the $\mathrm{T}_{4}$ emission spectrum (Figure 5 ) at $293 \mathrm{~K}$, and observed a rise-time of predominantly $130 \pm 12$ $\mathrm{fs}$, very close to the observed rate of EET in the dyad. We are therefore left to consider that such ultrafast structural relaxation, which is suggestive of planarization in the oligothiophene unit, is a downhill relaxation process that brings the excited state to a crossing point to enable transfer from oligothiophene to Bodipy.

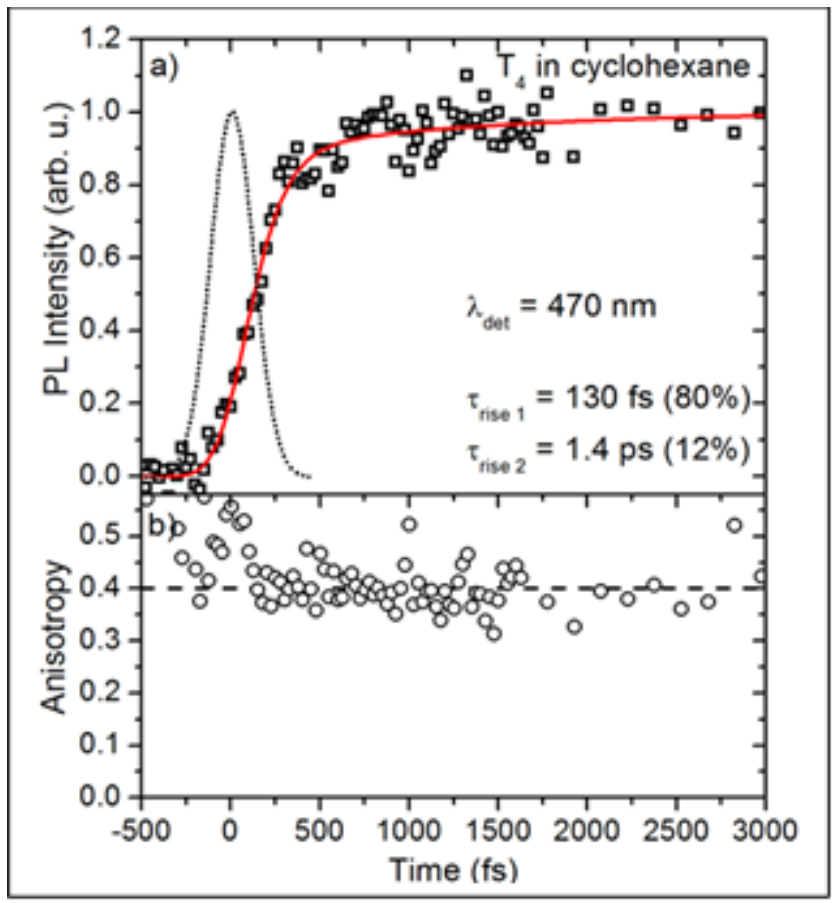

Figure 5. Fluorescence and its anisotropy kinetics for $\mathrm{T}_{4}$ when excited at $390 \mathrm{~nm}$. a) Detected magic angle emission at $470 \mathrm{~nm}$ (open squares). The solid line is a fit to the data, with rise-time constants of $130 \pm 12 \mathrm{fs}$ and $1.4 \pm 0.1 \mathrm{ps}$, found to fit best to the data with pre-exponential amplitudes shown in parenthesis in the inset. The IRF is shown as the dotted line. b) Fluorescence anisotropy kinetics at $470 \mathrm{~nm}$ (open circles), showing no depolarization of emission, with the anisotropy staying constant at its starting value of 0.4 \pm 0.01 (dashed line).

Strong electronic coupling can be evidenced by spectral changes in absorption of Bodipy when isolated or in the dyad. The absorption maxima for the Bodipy $S_{0}-S_{1}$ transition shifts from $525 \mathrm{~nm}$ in the isolated molecule to $543 \mathrm{~nm}$ in BOD-T4 (Figure 1 ) and $544 \mathrm{~nm}$ in BOD-T significant electronic mixing between the chromophores. In further pursuance of this effect, it is notable that in DFT calculations the HOMO is localized on Bodipy, but the LUMO centered on Bodipy spreads onto the first (and to a lesser extent the second) thiophene unit of BOD-T4 (Figure 6). The LUMO in BOD-T5 is more localized because of increased geometric distortion, but nonetheless there appears to be a small delocalization onto the first thiophene ring. More significantly, examination of the HOMO and LUMO primarily associated with the $\mathrm{T}_{4}$ unit of the dyad shows very clear indications for orbital mixing between the subunits for the LUMO but not the HOMO. Again, orbital mixing is less pronounced for BOD-T5 but does occur for the LUMO. Such delocalization introduces a certain degree of commonality into the $\mathrm{S}_{1}$ levels associated with Bodipy and oligothiophene.

We are thus left to consider a system that shows strong electronic coupling between the donor and acceptor as well as rapid structural relaxation on the donor with a time constant remarkably similar to that of the EET event itself. It is interesting to note that coherent electron-nuclear coupling has been observed in oligothiophene molecular wires. 35 This process, for which the main requirement is the coupling of two electronic levels whose energy spacing is comparable to one molecular vibration, leads to a breakdown of the Born-Oppenheimer approximation. As such, rapid structural relaxation of $\mathrm{T}_{4}$ (or $\mathrm{T}_{5}$ ) in the excited-state manifold is a reasonable process to consider. Consistent with this picture, it has been reported ${ }^{36}$ that vibrational coherence in oligothiophenes can persist for up to $500 \mathrm{fs}$. The ultrafast structural relaxation in the oligothiophene can bring the excited state of the $\mathrm{T}_{4}$ unit on the potential energy surface (PES) to a conical intersection with the Bodipy surface. Strong electronic coupling between the two surfaces will enable an efficient transfer of energy, aided by the shared transition density on the first thiophene unit between the two states. This process gives rise to EET that proceeds exceptionally quickly, despite the poor spectral overlap between the two chromophores and despite their near orthogonal orientation. This is important to consider in the design of light harvesting materials and systems where energy is required to be efficiently shuttled through space. Our work confirms ${ }^{8}$ the notion that strong spectral overlap is not a pre-requisite for fast EET, provided the excited-state processes are optimized. As such, dyads such as BOD-T4 may represent a future class of materials that move beyond weak-coupling to aid in applications that demand efficient energy transfer.

\section{CONCLUDING REMARKS}

This work reports exceptionally rapid EET with a time constant of 120 fs across an orthogonally-sited molecular dyad for which there is only a modest spectral overlap integral and for which there is a significant dipole reorientation of $64^{\circ}$. Our results show EET can be much faster than previous studies have observed on different perpendicularly held molecular dyads. 8,10 Other intriguing observations relate to the non-equilibrium geometry of the donor and the lack of correlation between rate of EET and degree of spectral overlap. It has been reported,37 however, that 
the strict requirement of spectral overlap between the donor emission spectrum and the acceptor absorption spectrum is relaxed if modes couple simultaneously to the donor and acceptor electronic excitations with opposite sign displacements. Orbital mixing of the LUMO, especially for the oligothiophene unit, is further testimony for shared occupancy and is perhaps the most critical point; certainly this effect is responsible for the red shifted absorption spectrum observed for the Bodipy chromophore in these dyads.

Our current understanding, therefore, is that excitation of the oligothiophene donor causes rapid structural relaxation that is inconsistent with the Born-Oppenheimer approximation. ${ }^{34}$ This structural relaxation, presumed to involve planarization, moves the excited state to a conical intersection on the potential energy surface between donor and acceptor, where EET occurs. The net result is ultrafast electronic energy transfer, for which the rate is independent of the spectral overlap term.

\section{SUPPORTING INFORMATION}

Supporting Information includes the following: auto-reconvolution data, depolarization fitting, anisotropy of BODIPY, formulae of control compounds, energy-minimized geometries, comparison of absorption / excitation spectra, Kohn-Sham representations, fluorescence spectra after excitation into oligothiophene at $77 \mathrm{~K}$ and RT. This material is available free of charge via the Internet at http://pubs.acs.org.

\section{AUTHOR INFORMATION}

\section{Corresponding Author}

*anthony.harriman@ncl.ac.uk. Tel: +44 1912088660.

\section{Author Contributions}

The manuscript was written through contributions of all authors.

\section{Funding Sources}

EPSRC : European Union Seventh Framework Programme.

\section{ACKNOWLEDGMENT}

This work was funded by EPSRC Grants EP/Go4094X/1 and

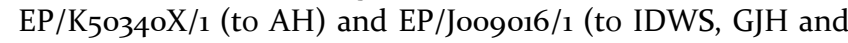
AR) and from the European Union Seventh Framework Programme under grant agreement 321305 (IDWS).

\section{REFERENCES}

(1) Panitchayangkoon, G.; Hayes, D.; Fransted, K. A.; Caram, J. R.; Harel, E.; Wen, J. Z.; Blankenship, R. E.; Engel, G. S. Long-lived quantum coherence in photosynthetic complexes at physiological temperatures. Proc. Natl. Acad. Sci. 2010, 107, 12766-12770.

(2) Middleton, C. T.; de la Harpe, K.; Su, C.; Law, Y. K.; CrespoHernandez, C. E.; Kohler, B. DNA Excited state dynamics: From single bases to the double helix. Ann. Rev. Phys. Chem. 20o9, 6o, 217-239.

(3) Fan, C. H.; Plaxco, K. W.; Heeger, A. J. Biosensors based on binding-modulated donor-acceptor distances. Trends Biotechnol. 2005, 23, 186-192.
(4) Beljonne, D.; Curutchet, C.; Scholes, G. D.; Silbey, R. J. Beyond Förster resonance energy transfer in biological and nanoscale systems. J. Phys. Chem. B 2009, 113, 6583-6599.

(5) Schwartz, B. Conjugated polymers as molecular materials: How chain conformation and film morphology influence energy transfer and interchain interactions. Ann. Rev. Phys. Chem. 2003, 54, 141-172.

(6) Hsu, C.-P.; Fleming, G. R.; Head-Gordon, M.; Head-Gordon, T. Excitation energy transfer in condensed media. J. Chem. Phys. 2001, 114, 3065-3072.

(7) Curutchet, C.; Mennucci, B.; Scholes, G. D.; Beljonne, D. Does Förster theory predict the rate of electronic energy transfer for a model dyad at low temperature? J. Phys. Chem. B 2008, 112, 3759-3766.

(8) Becker, K.; Lupton, J. M.; Feldmann, J.; Setayesh, S.; Grimsdale, A. C.; Müllen, K. Efficient intramolecular energy transfer in single end-capped conjugated polymer molecules in the absence of appreciable spectral overlap. J. Am. Chem. Soc. 2006, 128, 680681.

(9) Hayes, D.; Griffin, G. B.; Engel, G. S. Engineering coherence among excited states in synthetic heterodimer systems. Science 2013, 340, 1431-1434.

(10) Langhais, H.; Esterbauer, A. J.; Walter, A.; Riedle, E.; Puglies, I. Förster resonant energy transfer in orthogonally arranged chromophores. J. Am. Chem. Soc. 2010, 132, 16777-16782.

(11) Wong, K. F.; Bagchi, B.; Rossky, P. J. Distance and orientation dependence of electronic energy transfer rates in conjugated systems: Beyond the Förster theory. J. Phys. Chem. A 2006, 108, $5752-5763$.

(12) Harriman, A.; Mallon, L. J.; Elliot, K. J.; Haefele, A.; Ulrich, G.; Ziessel, R. Length dependence for intramolecular energy transfer in three- and four-color donor-spacer-acceptor arrays. J. Am. Chem. Soc. 2009, 131, 13375-13386.

(13) Engel, G. S.; Calhoun, T. R.; Read, E. L.; Ahn, T. K.; Mancal, T.; Cheng, Y. C.; Blankenship, R. E.; Fleming, G. R. Evidence for wave-like energy transfer through quantum coherence in photosynthetic systems. Nature 2007, 446, 782-786.

(14) Shaw, P. E.; Ruseckas, A.; Samuel, I. D. W. Distance dependence of excitation energy transfer between spacer-separated conjugated polymer films. Phys. Rev. B 2008, 78, 245201.

(15) Wu, P. G.; Brand, L. Resonance energy transfer - Methods and applications. Anal. Biochem. 1994, 218, 1-13.

(16) Scholes, G. D. Long-range resonance energy transfer in molecular systems. Ann. Rev. Phys. Chem. 2003, 54, 57-87.

(17) Renger, T. Theory of excitation energy transfer: From structure to function. Photosynth. Res. 2009, 102, 471-485.

(18) Ishizaki, A.; Fleming, G. R. On the adequacy of the Redfield equation and related approaches to the study of quantum dynamics in electronic energy transfer. J. Chem. Phys. 2oo9, 130, 234110.

(19) van Dantzig, N. A.; Levy, D. H.; Vigo, C.; Piotrowiak, P. Vibronic coupling and energy transfer in bichromophoric molecules. The effect of symmetry. J. Chem. Phys. 1995, 103, 4894-4899.

(20) Alamiry, M. A. H.; Hagon, J. P.; Harriman, A.; Bura, T.; Ziessel, R. Resolving the contribution due to Forster-type intramolecular electronic energy transfer in closely-coupled molecular dyads. Chem. Sci. 2012, 3, 1041-1048.

(21) Benniston, A. C.; Copley, G.; Harriman, A.; Rewinska, D. B.; Harrington, R. W.; Clegg, W. A donor-acceptor molecular dyad showing multiple electronic energy-transfer processes in crystalline and amorphous states. J. Am. Chem. Soc. 20o8, 130, 7174-7175.

(22) Hayes, G. R.; Samuel, I. D. W.; Phillips, R. T. Polarization dependence of the ultrafast photoluminescence of oriented poly(p-phenylenevinylene). Phys. Rev. B 1997, 56, 3838-3843. 
(23) Zade, S. S.; Bendikov, M. Twisting of conjugated oligomers and polymers: Case study of oligo- and polythiophene. Chem. Eur. J. 2007, 13, 3688-3700.

(24) Alamiry, M. A. H.; Hagon, J. P.; Harriman, A.; Bura, T.; Ziessel, R. Resolving the contribution due to Förster-type intramolecular electronic energy transfer in closely coupled molecular dyads. Chem. Sci. 2012, 3, 1041-1048.

(25) Becker, R. S.; Seixas de Melo, J.; Macanita, A. L.; Elisei, F. Comprehensive evaluation of the absorption, photophysical, energy transfer, structural, and theoretical properties of $\alpha$-oligothiophenes with one to seven rings. J. Phys. Chem. 1996, 100, 1868318695.

(26) Lakowicz, J. R. Principles of Fluorescence Spectroscopy, Third Edition (Springer, New York) 2006, Chapter 11, pp 383-412.

(27) Ameloot, M.; van de Ven, M.; Acuña, U.; Valeur, B. Fluorescence anisotropy measurements in solution: Methods and reference materials (IUPAC technical report). Pure Appl. Chem. 2013, $85,589-608$.

(28) Ruseckas, A.; Wood, P.; Samuel, I. D. W.; Webster, G. R.; Mitchell, W. J.; Burn, P. L. Ultrafast depolarization of the fluorescence in a conjugated polymer. Phys. Rev. B 2005, 72, 115214.

(29) Megow, J.; Röder, B.; Kulesza, A.; Bonačič-Koutecký, V.; May, V. A mixed quantum-classical description of excitation energy transfer in supramolecular complexes: Förster theory and beyond. ChemPhysChem 2011, 12, 645-656.

(30) Czikklely, V.; Forsterling, H. D.; Kuhn, H. Extended dipole model for aggregates of dye molecules. Chem. Phys. Lett. 1970, 6, 207-210.
(31) Sigal, H.; Markovitsi, D.; Gallos, L. K.; Argyrakis, P. Singlet excitation transfer in columnar liquid crystals studied by Monte Carlo simulation. J. Phys. Chem. 1996, 100, 10999-11004.

(32) Krueger, B. P.; Scholes, G. D.; Fleming, G. R. Calculation of couplings and energy transfer pathways between pigments of $\mathrm{LH}_{2}$ by the ab initio transition density cube method. J. Phys. Chem. B 1998, 102, 5378-5386.

(33) Casado, J.; Hicks, R. G.; Hernandez, V.; Myles, D. J. T.; Ruiz Delgado, M. C.; Lope Naverrette, J. T. Infrared and Raman features of a series of $\alpha, \omega$-bis(arylthio)oligothiophenes as molecular wires. A $\pi$-electron delocalization study. J Chem. Phys. 2003, 118, 1912-1920.

(34) Wong, K. S.; Wang, H.; Lanzani, G. Ultrafast excited-state planarization of the hexamethylsexithiophene oligomer studied by femtosecond time-resolved photoluminescence. Chem. Phys. Lett. 1988, 288, 59-64.

(35) Repp, J.; Liljeroth, P.; Meyer, G. Coherent electron-nuclear coupling in oligothiophene molecular wires. Nature Phys. 2010, 6 , 975-979.

(36) Du, J.; Wang, Z.; Feng, W.; Yoshino, K.; Kobayashi, T. Simultaneous measurement of electronic and vibrational dynamics to clarify a geometrical relaxation process in a conjugated polymer. Phys. Rev. B 2008, 77, 195205.

(37) Hennebicq, E.; Beljonne, D.; Curutchet, C.; Scholes, G. D.; Silbey, R. J. Shared-mode assisted resonant energy transfer in the weak coupling limit. J. Chem. Phys. 2009, 130, 214505. 


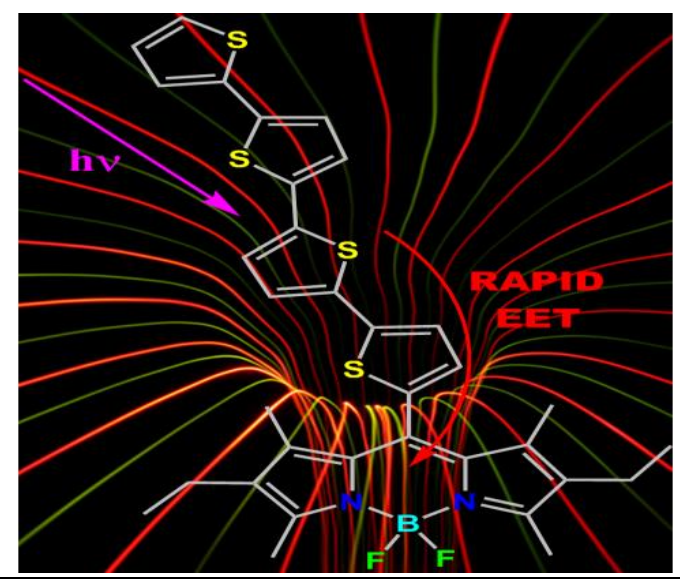

